\title{
Estrategias educativas para la motivación de los estudiantes del tercer año de bachillerato en el proceso de enseñanza-aprendizaje.
}

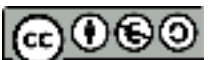

\section{Educational strategies for the motivation of students of the third year of bachelor's degree in the teaching-learning process.}

MSc. Ramiro Enrique Guamán Chávez ${ }^{1}$, Ing. Oswaldo Adrián Mendoza Antón. ${ }^{2}$, MSc. Peñafiel Villarreal Ruth Esther. ${ }^{3,}$ \& Est. Bone Peralta Piero Pastor. ${ }^{4}$

Recibido: 01-09-2017 / Revisado: 03-11-2017 Aceptado: 14-12-2017/ Publicado: 01-01-2018

\begin{abstract}
.
DOI: $\underline{\text { https://doi.org/10.33262/cienciadigital.v2i1.8 }}$

The purpose of this study, is to determine the impact that the use of educational strategies that allow the motivation of students in the third year of the Baccalaureate of the "Juan Montalvo" Educational Unit in the canton of La Concordia have for the teaching-learning process. The present is a non-experimental, descriptive, exploratory study. The statistical data supporting this research were obtained by processing the results of the survey application to 11 teachers, 37 students and 32 parents. The results show that there is a marked incidence for teaching learning the motivational strategies It is concluded that the low school performance of the students is in direct proportion with the use of strategies for motivation. Teachers do not know and do not master these strategies to encourage motivation and therefore the problem that affects the educational performance of students is presented.
\end{abstract}

Keywords: Estrategias Educativas, Motivación, Aprendizaje, Rendimiento Escolar.

\section{Resumen.}

La finalidad del presente estudio, es determinar la incidencia que tienen para el proceso de la enseñanza aprendizaje el uso de las estrategias educativas que permitan la motivación a los estudiantes del tercer año de Bachillerato de la Unidad

\footnotetext{
${ }^{1}$ Universidad Técnica Luis Vargas Torres Ext. La Concordia, Ecuador, ramiro.guaman@utelvt.edu.ec 2Universidad Técnica Luis Vargas Torres Ext. La Concordia, Ecuador, oswaldo.mendoza@utelvt.edu.ec ${ }^{3}$ Universidad Técnica Luis Vargas Torres Ext. La Concordia, Ecuador, ruthpenafielv@ hotmail.com ${ }^{4}$ Universidad Técnica Luis Vargas Torres Ext. La Concordia, Ecuador, boneperalta97@hotmail.com
} 
Educativa "Juan Montalvo" del cantón La Concordia. El presente es un estudio no experimental, descriptivo, exploratorio. Los datos estadísticos que sustentan esta investigación se obtuvieron procesando los resultados de la aplicación de encuestas a 11 docentes, 37 estudiantes y 32 padres de familia. Los resultados demuestran que existe una marcada incidencia para la enseñanza aprendizaje las estrategias motivacionales Se concluye que el bajo rendimiento escolar de los alumnos esta en proporción directa con el uso de estrategias para la motivación. Los docentes no conocen y no dominan estas estrategias para incentivar la motivación y por ello se presenta el problema que perjudica el rendimiento educativo de los estudiantes.

Palabras Claves: Estrategias educativas, motivación, aprendizaje, rendimiento escolar.

\section{Introducción.}

Las Estrategias Educativas para la Motivación de los estudiantes atienden no solamente factores del currículo de orden intelectual, sino también el desarrollo interpersonal, como parte esencial del aprendizaje. Es el mirar a la persona en el contexto interno y externo es lo que permite tanto a docentes y estudiantes interactuar con éxito en su preparación para el mundo real; aquel mundo que se recrea y crea permanentemente en la aulas sin muchas veces saber qué y para que se lo hace (Ruíz , 2009).

Este fenómeno responde justamente a que los docentes no han sido concienciados de la labor educativa que deben desarrollar para la toma de conciencia de sus estudiantes que se convertirán en los ciudadanos del mañana inmediato y que serán la muestra fehaciente de lo que han sido sus maestros en el compromiso de la formación integral de su personalidad para el desarrollo de sus potencialidades a fin de en forma individual o colectiva incidir en el desarrollo de los pueblos (Camposeco, 2012).

Los profesores en su mayoría desconocen las estrategias educativas para la motivación de los estudiantes (Guaman, 2017). Los padres de familia simplemente cumplen con enviar a sus hijos a los colegios mas no los motivan e incentivan a lograr conocimientos nuevos y eficaces para su formación personal, Las autoridades del plantel no se han preocupado de hacer un seguimiento y monitoreo de las causas que generan la apatía y el desinterés de los estudiantes para lograr los aprendizajes (García \& Doménech Bet, 2014). La supervisión educativa no proporciona cursos y/o seminarios a los docentes sobre la motivación como fuente generadora de interés para el proceso de enseñanza aprendizaje de los estudiantes. Los estudiantes encuentran fuentes de motivación ajenas a la educación en lugares del entorno socio económico y descuidan los aprendizajes escolarizados (García \& Doménech Bet, 2014). Los intereses de los estudiantes se desvían a la adquisición de bienes económicos y materiales más descuidan sus tareas escolares (TARGET, 2005). 
Uno de los focos generadores de la desmotivación estudiantil se refleja en la poca afectividad que los docentes aplican a sus estudiantes, olvidando que muchos de ellos tienen problemas emocionales derivados de la constitución de sus hogares los mismos que por efecto de la migración sufren sus consecuencias. Basta preguntar a los jóvenes si viven con sus padres y madres para confirmar esta aseveración, si los estudiantes del tercer año de bachillerato no son motivados e incentivados a través de proceso psicopedagógicos para adquirir los conocimientos básicos de la enseñanza aprendizaje, su rendimiento académico será insipiente, se privaran de las oportunidades de adquirir conocimientos, habilidades y destrezas mentales que los impela a mejorar su grado cultural y su accionar consciente en el entorno social en donde se desarrollan (Guaman,2017).

Al fomentar el desarrollo de las Estrategias Educativas para la Motivación en los estudiantes del tercer años de bachillerato, se generara un cambio de actitud, se mejorara el rendimiento académico, se mejorará el comportamiento emocional y la auto estima de los estudiantes (Tapia , 2007). Lamentablemente los cursos y seminarios que ha proporcionado el Ministerio de Educación poco o nunca tratan de proporcionar estratégicas educativas que generen estrategias educativas para la motivación de los aprendientes y si lo hacen, es de una forma superficial que no va más allá de la conceptualización (MINEDUC, 2015). Por ello se aprecia tanto en los docentes como en los estudiantes un notable desinterés hacia el acto educativo, el mismo que resulta como una obligación para los aprendientes y una justificación para los padres de familia en decir que le han dado a sus hijos la oportunidad de asistir a la unidad educativa, como lo reza la constitución de la república del Ecuador (Asamblea Nacional del Ecuador , 2008), sin importar que estos realmente a su egreso estén procesos de conocimientos, que les conduzca a la liberación conscientes de sus actos y a lo propensión de nuevas oportunidades de trabajo y de estudio por que realmente se encuentren motivados a ello (Ruíz , 2009).

Los docentes de la Unidad Educativa Juan Montalvo tienen estas falencias por ello sus estudiantes son poco afectos al proceso de aprendizaje pero, también es ciertos que estos docentes están ávidos de conocer más a fondo los procesos de la motivación para incidir positivamente en el rendimiento académico de los estudiantes y poder cumplir conscientemente con la tarea de formar las personalidades del futuro para el desarrollo de la comunidad y del país (Guaman, Benitez, \& Ramirez, 2017).

Determinar las causas que impiden la motivación positiva en los estudiantes de la unidad educativa Juan Montalvo del Cantón La Concordia, con el fin de generar estrategias como alternativas de mejoras en el rendimiento académico de los estudiantes del tercer año de bachillerato, e incidir positivamente a la obtención dinámica, activa, consciente y efectiva para lograr los objetivos de la educación de calidad (Guaman \& Cevallos, 2016).

\section{Métodos y materiales.}


Este trabajo está diseñado de acuerdo con las modalidades de investigación de campo, con una muestra de 11 docentes, 37 estudiantes y 32 padres de familia, para lograr bibliográfica-documental, experimental mediante el método el exploratorio-descriptivo un resultado apegado a la realidad y considerando el tamaño de la población no se realizó ningún tipo de muestreo y se consideró a todo el universo para el respectivo análisis. Además se utilizó una encuesta para la recolección de la información, así mismo se trasladaron los datos recopilados a una matriz de tabulación elaborada previamente por el ordenador. Después del análisis e interpretación de los resultados, se elaboraron los cuadros y gráficos estadísticos, para comprobar los objetivos planteados que llevaron a la investigación.

\section{Análisis y discusión de resultados.}

Tabla1. ¿Cuánto te agradan las clases que recibes de tus profesores?

\begin{tabular}{lcc}
\hline Alternativa & F. & \% \\
\hline Mucho & 6 & $17 \%$ \\
Poco & 12 & $33 \%$ \\
Nada & 19 & $50 \%$ \\
Total & $\mathbf{3 7}$ & $\mathbf{1 0 0 \%}$ \\
\hline
\end{tabular}

Elaborado por: Ramiro Guamán.

El $50 \%$ de los estudiantes no les agrada la forma como sus profesores dan las clases y esta respuesta tácitamente dice a la aprehensión de conocimientos para lograr un rendimiento académico favorable.

Tabla2. Cuando el profesor va a dar una clase nueva, dice a ustedes para qué sirve el tema y la clase que dará?

\begin{tabular}{lcc}
\hline \multicolumn{1}{c}{ Alternativa } & F. & \% \\
\hline Siempre & 7 & $20 \%$ \\
A veces & 14 & $37 \%$ \\
Nunca & 16 & $43 \%$ \\
Total & $\mathbf{7 0}$ & $\mathbf{1 0 0 \%}$ \\
\hline
\end{tabular}

Elaborado por: Ramiro Guamán.

En un $43 \%$ las clases que imparte el maestro no les gusta a los estudiantes por cuanto no están motivados para adquirir nuevos conocimientos; no se valoriza el contenido ni se lo relaciona con los intereses de los estudiantes. Simplemente se da la clase sin que ellos realmente la acojan por la utilidad que presta para su profesión. 
Tabla3. ¿Su profesor le pregunto a usted que quiere ser y le indico lo que debe hacer, para lograr lo que usted quiere ser?

\begin{tabular}{lcc}
\hline Alternativa & F. & \% \\
\hline $\mathrm{Si}$ & 8 & $21 \%$ \\
$\mathrm{No}$ & 29 & $79 \%$ \\
Total & $\mathbf{3 7}$ & $\mathbf{1 0 0 \%}$ \\
\hline
\end{tabular}

Elaborado por: Ramiro Guamán.

Los docentes no estimulan a los estudio para lograr ser personas prestantes a la sociedad. Tampoco explican cómo conseguir los propósitos de una profesión u oficio.

Tabla4. ¿Realiza su profesor clases interesantes?

\begin{tabular}{lcc}
\hline Alternativa & F. & $\mathbf{\%}$ \\
\hline Muy interesantes & 9 & 24 \\
No interesantes & 28 & 76 \\
Total & $\mathbf{3 7}$ & $\mathbf{1 0 0 \%}$ \\
\hline
\end{tabular}

Elaborado por: Ramiro Guamán.

La falta motivación por parte de los docentes implica un desinterés por los estudiantes implicando un bajo nivel educativo.

Tabla 1. ¿Qué le gusta aprender más?

\begin{tabular}{lcc}
\hline Alternativa & F. & \% \\
\hline Cosas practicas & 15 & $40 \%$ \\
Cosas que lo hacen pensar & 17 & $46 \%$ \\
No le gustan ninguna de las & 5 & $14 \%$ \\
dos & & \\
Total & $\mathbf{3 7}$ & $\mathbf{1 0 0 \%}$ \\
\hline
\end{tabular}

Elaborado por: Ramiro Guamán.

Las preferencias de aprendizajes permiten que el docente tenga un conocimiento de los intereses e individualidades de los estudiantes por lo tanto no existe el grado homogéneo.

Tabla6. ¿Su maestro se preocupa cuando los estudiantes no aprenden las clases? 
Elaborado por: Ramiro Guamán.

\begin{tabular}{lll}
\hline Alternativa & F. & \% \\
\hline Siempre & 7 & $20 \%$ \\
A veces & 12 & $33 \%$ \\
Nunca & 17 & $47 \%$ \\
Total & $\mathbf{3 7}$ & $\mathbf{1 0 0 \%}$ \\
\hline
\end{tabular}

Los alumnos son muy perceptivos y las respuestas dadas por los estudiantes deben cambiar la forma de impartir las clases y las acciones de estimular que genera en sus estudiantes.

Tabla7. Usted se siente más a gusto cuando el profesor le pide:

\begin{tabular}{lcc}
\hline Alternativa & F. & \% \\
\hline $\begin{array}{l}\text { Realizar una actividad que te gusta y da } \\
\text { placer }\end{array}$ & 16 & $43 \%$ \\
Investigar o tratar de entender algo nuevo & 19 & $50 \%$ \\
Cuando te obligan a que hagas algo & 3 & $7 \%$ \\
Total & $\mathbf{3 7}$ & $\mathbf{1 0 0 \%}$ \\
\hline
\end{tabular}

Elaborado por: Ramiro Guamán.

El $50 \%$ de los estudiantes comentan que se sienten muy a gusto cuando se les permite investigar o tratar de entender algo nuevo. Estas respuestas deben de servirle al docente como parámetros para el desarrollo de sus clases. El porcentaje minoritario obedece sin lugar a dudas a aquellos estudiantes no emotivos.

Tabla8. ¿Sus profesores le permiten realizar actividades que te gusta y te dan placer?

\begin{tabular}{lcc}
\hline \multicolumn{1}{c}{ Alternativa } & F. & \% \\
\hline Siempre & 5 & $14 \%$ \\
A veces & 13 & $36 \%$ \\
Nunca & 19 & $50 \%$ \\
Total & $\mathbf{3 7}$ & $\mathbf{1 0 0 \%}$ \\
\hline
\end{tabular}

Elaborado por: Ramiro Guamán.

Al preguntar a los estudiantes si los profesores le permiten realizar actividades que les gustan y les dan placer el $50 \%$ dijo que nunca; el 36\% dice que solo a veces y, $14 \%$ dice que siempre. Aquí se aprecia las pocas oportunidades que dan los educadores a sus estudiantes a realizar actividades que coordinadas con la educación le place realizar a los estudiantes. 
Tabla 2. ¿Sus profesores le permiten que usted investigue o trate de entender algo nuevo?

\begin{tabular}{lcc}
\hline \multicolumn{1}{c}{ Alternativa } & F. & \% \\
\hline Siempre & 5 & $13 \%$ \\
A veces & 14 & $38 \%$ \\
Nunca & 18 & $49 \%$ \\
Total & $\mathbf{7}$ & $\mathbf{1 0 0 \%}$ \\
\hline
\end{tabular}

Elaborado por: Ramiro Guamán.

De la investigación realizada el $87 \%$ de los estudiantes están impedidos de realizar investigaciones o traten de entender algo que les gusta mucho.

Tabla 3. ¿Cuál de las siguientes situaciones son más ajustadas a usted:

\begin{tabular}{lcc}
\hline \multicolumn{1}{c}{ Alternativa } & F. & \% \\
\hline $\begin{array}{l}\text { Estudio la noche antes del examen porque } \\
\text { mis padres me fuerzan a hacerlo }\end{array}$ & 23 & $61 \%$ \\
$\begin{array}{l}\text { Estudié para este examen porque el examen } \\
\text { anterior lo reprobé por no estudiar". }\end{array}$ & 11 & $29 \%$ \\
$\begin{array}{l}\text { Decidí estudiar anoche porque es algo } \\
\text { importante para mí }\end{array}$ & 4 & $10 \%$ \\
Total & $\mathbf{3 7}$ & $\mathbf{1 0 0 \%}$ \\
\hline
\end{tabular}

Elaborado por: Ramiro Guamán

Considera los estudiantes que mayoritariamente los motivos para estudiar lo encuentran en una obligación impuesta, es decir no hay una motivación intrínseca o propia y esto se debe con seguridad a que los profesores no estimulan esta condición

Tabla 4. ¿Cómo son tus padres cuando no llevas buenas notas?

\begin{tabular}{lcc}
\hline \multicolumn{1}{c}{ Alternativas } & F. & \% \\
\hline Castigadores e insultantes & 8 & $21 \%$ \\
Aconsejadores y razonables & 6 & $17 \%$
\end{tabular}


No le prestan atención al problema de rendimiento

Elaborado por: Ramiro Guamán.

De la investigación se puede observar la importancia de los padres y la actitud frente al hecho educativo. La parte psicológica inciden en la motivación de los estudiantes frente al hecho educativo y relacionando con la actitud de los docentes frente a la motivación, se establece que ellos deben ser los concienciadores de los intereses y motivaciones de los estudiantes por lo que deben mejorar sus técnicas de enseñanza aprendizaje y brindar las oportunidades procesualmente hasta lograr que los niños y niñas encuentren realmente los motivos para su aprendizaje.

Tabla 5. ¿Sus profesores y sus padres de familia le permiten a usted que arme y desarme objetos, juguetes, rompecabezas, cosas viejas, etc.?

\begin{tabular}{lcc}
\hline \multicolumn{1}{c}{ Alternativas } & F. & \% \\
\hline $\mathrm{Si}$ & 7 & $20 \%$ \\
No & 30 & $80 \%$ \\
Total & $\mathbf{3 7}$ & $\mathbf{1 0 0 \%}$ \\
\hline
\end{tabular}

Elaborado por: Ramiro Guamán.

La curiosidad es una situación innata, los padres de familia y los docentes deben recordar sus tiempos de niños y encontrar en ese pasado lo que es ahora el presente de los niños, por tanto deben en vez de reprimir, deben permitir que los niños encuentren una explicación al por qué y al cómo funcionan las cosas. Ello le permitirá integrarse al mundo de la mecánica y de las ciencias y tendrá argumentos de que hablar con sus amigos y compañeros, le permitirá inclusive elementos de juicio para la creatividad por cuanto están motivados.

\section{Resultados de las encuestas a los padres de familia}

Tabla 6. ¿Usted como padre le ha preguntado a su hijo que quiere ser y le ha indicado lo que debe hacer para lograrlo? 


\begin{tabular}{llc}
\hline \multicolumn{1}{c}{ Alternativa } & F. & \% \\
\hline Si & 10 & $27 \%$ \\
No & 27 & $73 \%$ \\
Total & $\mathbf{3 7}$ & $\mathbf{1 0 0 \%}$ \\
\hline
\end{tabular}

Elaborado por: Ramiro Guamán.

El 73\% de los padres de familia no le han preguntado a su hijo que quiere ser y no les han Indicado lo que deben hacer para lograr lo que de mayor quiere ser El 27\% en cambio sí lo han hecho. Aquí se aprecia la falta de comunicación por causas diversas entre padres e hijo; se aprecia igualmente que ni siquiera los propios padres saben lo que quieren que más tarde sean sus hijos es decir no tienen un norte definido y consecuentemente el futuro de los hijos está en el quesea lo que puedan ser. Esta es una tarea importante que deben asumir los profesores en lo que a orientación a padres de familia deben dar para que ellos en conciencia puedan también descubrir, orientar y dar posibilidades a sus hijos para lo que serán mañana.

Tabla 7. Qué es lo que a su hijo le gusta aprender más.

\begin{tabular}{lcc}
\hline \multicolumn{1}{c}{ Alternativa } & F. & \% \\
\hline Cosas prácticas & 7 & $22 \%$ \\
Cosas que lo hacen pensar & 11 & $33 \%$ \\
No le gustan ninguna de las & 14 & $45 \%$ \\
dos & $\mathbf{3 2}$ & $\mathbf{1 0 0 \%}$ \\
\hline
\end{tabular}

Elaborado por: Ramiro Guamán.

El 45\% de los padres de familia investigados sostienen que a sus hijos no les gusta no aprender cosas prácticas ni cosas que los hagan pensar; el 33\% dice que a sus hijos les interesan las cosas que los hacen pensar y el $22 \%$ manifestaron que a sus chicos les interés las cosas prácticas. Estas apreciaciones de los padres parecen ser muy a priori puesto que contrastan con la de los propios estudiantes. 
Tabla 8. ¿Ustedes como padres de familia permiten a sus hijos realizar actividades que les gusta y que le dan placer?

\begin{tabular}{lcc}
\hline \multicolumn{1}{c}{ Alternativa } & F. & \% \\
\hline Siempre & 6 & $20 \%$ \\
A veces & 12 & $37 \%$ \\
Nunca & 14 & $43 \%$ \\
Total & $\mathbf{3 2}$ & $\mathbf{1 0 0 \%}$ \\
\hline
\end{tabular}

Elaborado por: Ramiro Guamán.

El $80 \%$ de los padres deben comprender que estas actividades y los juegos son parte de la estructuración de los comportamientos y el desarrollo de la inteligencia sin atrofiarla con castigos y represiones. Estas actividades que desarrollan los menores es justamente debido a su motivación intrínseca y no es bueno coártala sino controlarla e incentivarla para que la misma crezca y se fortalezca en el niño.

Tabla 9. ¿Usted como padre de familia les permiten a sus hijos que usted Investiguen o traten de entender algo nuevo?

\begin{tabular}{lcc}
\hline \multicolumn{1}{c}{ Alternativa } & F. & \% \\
\hline Si & 12 & $36 \%$ \\
No & 20 & $64 \%$ \\
Total & $\mathbf{3 2}$ & $\mathbf{1 0 0 \%}$ \\
\hline
\end{tabular}

Elaborado por: Ramiro Guamán.

De esta investigación se concluye que existen padres chapados a la antigua, muy autoritarios y por tanto están negando la posibilidad de que sus hijos se auto motiven hacia la búsqueda de nuevos conocimientos esto se debe posiblemente a lagunas frustraciones que ellos tuvieron en su infancia o al propio sistema de crianza del hogar (clase de líder del hogar). En todo caso los padres de familia deben cambiar de criterios en razón del desarrollo de la sociedad y de la ciencia y tecnología por el mañana, seguro y promisorio de sus hijos. 
Tabla 10. ¿Cuál de las siguientes situaciones Ud. realiza frente a los estudios de su hijo?

\begin{tabular}{lcc}
\hline \multicolumn{1}{c}{ Alternativa } & F. & \% \\
\hline $\begin{array}{l}\text { Esforzar a sus hijos a estudiar la noche } \\
\text { antes del examen }\end{array}$ & 10 & $32 \%$ \\
$\begin{array}{l}\text { Que el estudiante determine si lo hace } \\
\text { porque es algo importante para él }\end{array}$ & 22 & $68 \%$ \\
Total & $\mathbf{3 2}$ & $\mathbf{1 0 0 \%}$ \\
\hline
\end{tabular}

\section{Elaborado por: Ramiro Guamán.}

El recordar las obligaciones de los hijos no es malo, pero sí el obligarlo a estudiar la noche antes del examen cuando pudo hacerlo durante todo el siclo académicos esto refleja un porcentaje del $68 \%$. En una noche no podrá recuperar lo del trimestre o lo del año.

Tabla 11. ¿Cómo es su comportamiento cuando su hijo no llevas buenas notas?

\begin{tabular}{lcc}
\hline \multicolumn{1}{c}{ Alternativas } & F. & \% \\
\hline Castigador e insultante & 7 & $21 \%$ \\
Aconsejador y razonable & 14 & $45 \%$ \\
No le prestan atención al problema & 11 & $34 \%$ \\
de rendimiento & $\mathbf{3 2}$ & $\mathbf{1 0 0 \%}$ \\
Total &
\end{tabular}

Elaborado por: Ramiro Guamán.

Al realizar una encuesta que tocan la susceptibilidad de los encuestados estos se ponen a la defensiva y tratan de razonar su respuesta de manera que pierde la sinceridad y desmejora la credibilidad. La actitud de los que no prestan atención a los problemas de la educación de sus hijos a más de los problemas citados de la autoestima y los complejos de inferioridad, generan resentimiento e irrespeto hacia los padres que no se preocupan de su rendimiento y de su vida.

Tabla 12. ¿Si Ud. le prestara más atención a los problemas y necesidades de sus hijos ellos estarían motivados a lograr un mejor rendimiento escolar? 


\begin{tabular}{lcc}
\hline \multicolumn{1}{c}{ Alternativa } & F. & \% \\
\hline Si & 15 & $48 \%$ \\
No & 10 & $31 \%$ \\
Tal vez & 7 & $21 \%$ \\
Total & $\mathbf{3 2}$ & $\mathbf{1 0 0 \%}$ \\
\hline
\end{tabular}

Elaborado por: Ramiro Guamán

Despertar la conciencia a los padres de familia sobre el rol que están desempeñando y el que deben desempeñar en la formación de sus hijos.

\section{Resultados de las encuestas a los profesores.}

Tabla 13. ¿Usted ha preguntado a sus alumnos qué quieren ser en el futuro y les ha indicado lo que deben hacer para lograrlo?

\begin{tabular}{lcc}
\hline \multicolumn{1}{r}{ Alternativa } & F. & \% \\
\hline Si & 4 & $38 \%$ \\
No & 7 & $62 \%$ \\
Total & $\mathbf{1 1}$ & $\mathbf{1 0 0 \%}$ \\
\hline
\end{tabular}

Elaborado por: Ramiro Guamán.

La investigación indica que los maestros realmente dan sus clases por darlas, sin señalar la finalidad que la misma persigue, no orientan los intereses y vocaciones de los estudiantes, no lo motivan a ser un profesional futuro con éxitos, simplemente que "sean lo que puedan ser según la suerte o el destino".

Tabla 14. ¿Sus estudiantes están motivados al estudio y al aprendizaje?

\begin{tabular}{lcc}
\hline \multicolumn{1}{c}{ Alternativa } & F. & \% \\
\hline $\mathrm{Si}$ & 3 & $23 \%$ \\
$\mathrm{No}$ & 8 & $77 \%$ \\
Total & $\mathbf{6 7}$ & $\mathbf{1 0 0 \%}$ \\
\hline
\end{tabular}

Elaborado por: Ramiro Guamán.

Los docentes mayoritariamente son conscientes de que los estudiantes no están motivados al estudio y a los aprendizajes escolarizados y, esto da una pauta para averiguar las causas de su desmotivación las mismas que se averiguan en la pregunta siguiente. 
Tabla 15. ¿Cuáles de las siguientes alternativas son las causas para que los alumnos no estén motivados al estudio y al aprendizaje?

\begin{tabular}{lll} 
Alternativa & F. & \% \\
\hline Hiperactivos y desatentos & 2 & $20 \%$ \\
Falta de intereses & 2 & $20 \%$ \\
Hogares con bajo nivel cultural & 3 & $31 \%$ \\
Castigo frecuentemente por los padres & 3 & $27 \%$ \\
Maestros no conocen los intereses de los niños & 0 & $0 \%$ \\
Maestros no aplican estrategias para la & 1 & $9 \%$ \\
motivación & & \\
Total & $\mathbf{1 1}$ & $\mathbf{1 0 0 \%}$ \\
\hline
\end{tabular}

Elaborado por: Ramiro Guamán.

Los docentes no son honestos en lo referente a juzgarse o autoevaluar su responsabilidad en la formación de los estudiantes cuando responden con cero porcentajes a las dos últimas alternativas. "la culpa nunca es nuestra".. ; Sin embargo, la culpa la encuentran en las otras alternativas, pero en lo que hace referencia a la falta de interés de los niños (31\%) justamente los investigados no se dan cuenta que esta es una responsabilidad que deben ellos la deben asumir puesto que sociedad le ha encomendado la formación de sus hijos. son demasiado amables par encontrar justificaciones en el "yo no soy culpable .. el otro es." A su vez la repuestas dan muestra de que los docentes no planifican sus clases en razón de los interese y necesidades de los estudiantes.

Tabla 16. ¿Qué actitudes asume usted para motivar y estimular a sus alumnos al estudio?

ALTERNATIVA

f. $\%$ 
Hiperactivos y desatentos

Falta de intereses

Hogares con bajo nivel cultural

Castigo frecuentemente por los padres

Maestros no conocen los intereses de los niños

Maestros no aplican estrategias para la motivación

TOTAL
$20 \%$

$20 \%$

$3 \quad 31 \%$

$327 \%$

$0 \%$

$1 \quad 9 \%$

11

$100 \%$

Elaborado por: Ramiro Guamán.

La actividad estimula cuando el alumno experimenta y descubre sus propias soluciones y esto es lo que debe hacer el docente: guiar, orientar y estimular a la producción de soluciones que luego se conviertan en motivación intrínseca para el estudiante.

Tabla 17. ¿Ud. conoce estrategias y técnicas de motivación?

\begin{tabular}{lcc}
\hline \multicolumn{1}{r}{ Alternativa } & F. & \% \\
\hline $\mathrm{Si}$ & 2 & $18 \%$ \\
$\mathrm{No}$ & 9 & $82 \%$ \\
Total & $\mathbf{1 1}$ & $\mathbf{1 0 0 \%}$ \\
\hline
\end{tabular}

Elaborado por: Ramiro Guamán.

El $85 \%$ de los maestros encuestados dicen no conocer de técnicas y estrategias de motivación, en cambio el $15 \%$ dice si conocer las estrategias y técnicas de motivación.

Tabla 18. ¿Le gustaría asistir a seminarios y talleres sobre estrategias y técnicas de motivación?

\begin{tabular}{lcc}
\hline \multicolumn{1}{c}{ Alternativa } & F. & $\boldsymbol{\%}$ \\
\hline $\mathrm{Si}$ & 11 & $100 \%$ \\
$\mathrm{No}$ & 0 & $0 \%$ \\
Total & $\mathbf{1 1}$ & $\mathbf{1 0 0 \%}$ \\
\hline
\end{tabular}

Elaborado por: Ramiro Guamán.

El 100\% de los maestros están predispuestos a asistir a seminario talleres de motivación. La sabia decisión repercutirá en los estudiantes en tanto y cuanto los docentes sean capaces de vivencial y poner en práctica los conocimientos que logren de los talleres de motivación. 


\section{Conclusiones.}

- A los estudiantes poco les agradan las clases que reciben de sus profesores; no son motivados e incentivados a la adquisición de conocimientos; no se valoriza el contenido y el mismo no se lo relaciona con los intereses de los estudiantes.

- Los docentes no estimulan a sus estudiantes hacia la consecución de metras y objetivos a través del estudio para lograr ser personas prestantes a la sociedad. Tampoco explican cómo conseguir los propósitos de una profesión u oficio.

- Los maestros muy poco se preocupan porque los alumnos no aprenden las clases, Los alumnos se sienten a gusto cuando se les permite investigar o tratar de entender algo nuevo; o, cuando se le permite realizar actividades que les gusta y les da placer

- Maestros dan pocas oportunidades a sus estudiantes a realizar actividades que coordinadas con la educación les placa realizar es decir, no le dan oportunidades de realizar investigaciones y experimentaciones cuando quieran entender algo que les gusta mucho.

- A los estudiantes se los obliga e imponen normas y metas prescindiendo de la motivación intrínseca o propia sin que se estimule esta condición, algunos estudiantes provienen de hogares cuyos padres son castigadores e insultantes cuando no llevan buenas notas. Estas situaciones de carácter psicológico inciden en la desmotivación de los estudiantes frente al hecho educativo.

- Docentes y padres de familia no permiten que los niños encuentren una explicación al por qué y al cómo funcionan las cosas. Y esto les impide integrarse al mundo de la mecánica y de las ciencias y tener argumentos (socio lingüístico) para la interrelación con sus amigos y compañeros, que le permitirá inclusive elementos de juicio para la creatividad por cuanto están motivados intrínsecamente.

- Padres de familia castigadores y autoritarios replican su sistema de crianza y no se sitúan en el momento social en el que se encuentran sus hijos, padres de familia, docentes y estudiantes tratan de que se estudie en las noches anteriores a rendir un examen y el resto del tiempo pasa desapercibido sabiendo que no podrá recuperar lo del trimestre o lo del año.

- Docentes sostienen que la desmotivación de los niños se deben a que provienen de hogares con bajo nivel cultural; a la falta de interés; son castigados frecuentemente por sus padres y un bajo porcentaje que obedece a la hiperactividad y la falta de atención de los niños Los docentes no son demasiado amables para encontrar justificaciones en el "yo no soy culpable, el otro es". Los maestros dicen no conocer de técnicas y estrategias de motivación y están predispuestos a asistir a seminario talleres de motivación. 


\section{Referencias bibliográficas.}

Alonso, J. (2011). Psicologia. Madrid: Mc. Graw Hill. Obtenido de http://spain-s3-mheprod.s3-website-eu-west-1.amazonaws.com/bcv/guide/capitulo/8448609190.pdf

Asamblea Nacional del Ecuador . (13 de julio de 2008). Constitucion de La Republica del Ecuador 2008 . Obtenido de Lexis : http://www.oas.org/juridico/pdfs/mesicic4_ecu_const.PDF

Camposeco , F. (2012). La autoeficacia como variable en la motivación intrínseca y extrínseca en matemáticas a través de un criterio étnico. Tesis Universidad Complutense de Madrid, 252. Madrid, España. Obtenido de http://eprints.ucm.es/16670/1/T34002.pdf

García , F., \& Doménech Bet, F. (2014). Motivación, aprendizaje y rendimiento escolar. Revista electronica de Motivación y Emosión, 1(0). Obtenido de http://reme.uji.es/articulos/pa0001/texto.html

Guaman , R., \& Cevallos , G. (2016). Análisis comparativo de estilos de aprendizaje y sus niveles de preferencia. Revista: Atlante. Cuadernos de Educación y Desarrollo. Obtenido de http://www.eumed.net/rev/atlante/2016/02/estilos.html

Guaman , R., Benitez , J., \& Ramirez, V. (2017). Aplicación de Software Educativo para Simulación, para Bachillerato Técnico en mecánica de las universidades educativas de modalidad semi-presencial. Rev. Cs. Doc, 3(3), 30-46. Obtenido de http://www.cienciasdeladocumentacion.cl/gallery/2\%20v3n4\%202017\%20csdoc.pd $\mathrm{f}$

Guaman, R. (2017). Formación docente en tribus juveniles urbanas para una educación del Sumak Kawsay. Inclusiones, 4, 113-123. Obtenido de http://www.revistainclusiones.com/gallery/7\%20v4\%20n\%20esp\%20jul\%20sep\%2 02017\%20rv\%20inc.pdf

Huertas, A. (1997). Motivanción querer es aprender. Argentina: Aique SA. Obtenido de http://www.terras.edu.ar/biblioteca/6/TA_Huertas_Unidad_4.pdf

Márquez , M., \& Abundez , M. (2015). La motivación en el aula: estrategia esencial para mejorar el aprendizaje en la escuela primaria. Revista Atlante cuaderno de educacion y desarrollo. Obtenido de http://atlante.eumed.net/motivacion-aula/

MINEDUC. (abril de 2015). Ley Orgánica de Educación Intercultural. Obtenido de Ministerio de Educacion del Ecuador : https://educacion.gob.ec/wpcontent/uploads/downloads/2017/02/Ley_Organica_de_Educacion_Intercultural_L OEI_codificado.pdf 
Ruíz , B. (2009). la Motivación en el aula funciones del profesor para mejorar la motivacion en el aprendizaje. Revista Innovación y Experiencia Educativas(15), 9. Obtenido de https://archivos.csif.es/archivos/andalucia/ensenanza/revistas/csicsif/revista/pdf/Nu mero_15/BELEN_NAVARRETE_1.pdf

Tapia , A. (2007). Motivar para el aprendizaje. Reseña educativa, 251. Obtenido de http://sohs.pbs.uam.es/webjesus/motiv_ev_autorr/motivar_escuela.pdf

TARGET. (2005). Dimensiones de la intervención motivacional en el aula: los Grupos la Evaluación y el Tiempo (GET). Obtenido de Utecnologia: http://www.utecnologica.org/tportal/portales/tp4964b0e1bk102/uploadImg/File/Teo riaPractEns/motivacion/4_modeloTARGET.pdf

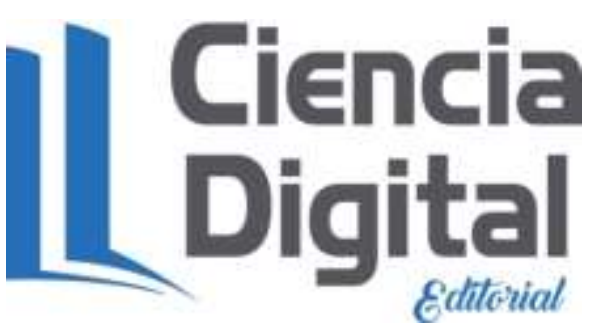

Para citar el artículo indexado. 
Guamán R., Mendoza O., Peñafiel R. y Bone P. (2018). Estrategias educativas para la motivación de los estudiantes del tercer año de bachillerato en el proceso de enseñanzaaprendizaje. Revista Electrónica Ciencia Digital 2(1), 105-121. Recuperado desde: http://www.cienciadigital.org/revistascienciadigital/index.php/CienciaDigital/article/view/8 18

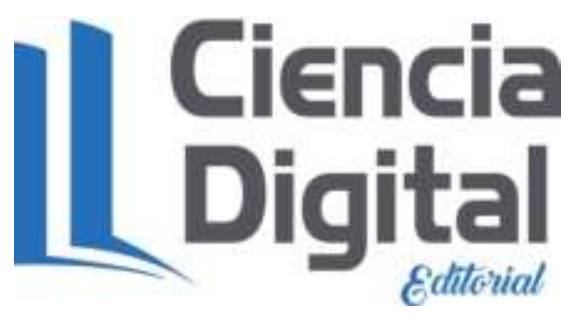

El artículo que se publica es de exclusiva responsabilidad de los autores y no necesariamente reflejan el pensamiento de la Revista Ciencia Digital.

El articulo queda en propiedad de la revista y, por tanto, su publicación parcial y/o total en otro medio tiene que ser autorizado por el director de la Revista Ciencia Digital.
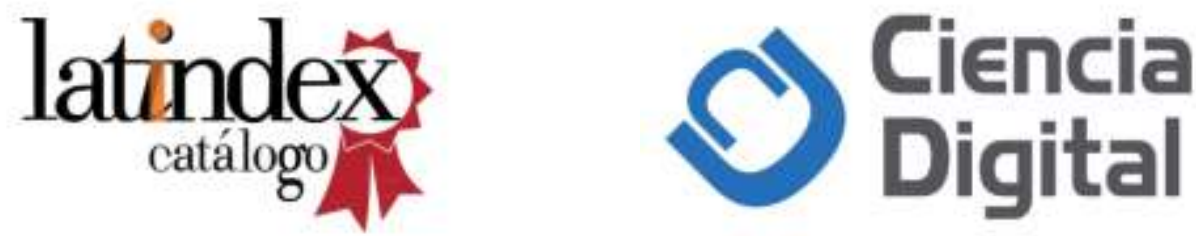